\title{
CINÉTICA DE SECAGEM DE HORTALIÇAS EM ESTUFA DE SECAGEM COM LUZ
}

\author{
B. M. TRAVÁliA ${ }^{1}$, B. S. DOS SANTOS ${ }^{1}$, L. G. M. $\operatorname{RESENDE}^{1}$, A. R. S. TELES ${ }^{1}$, L. C. L. A. \\ SANTANA $^{1}$, J. A. B. DOS SANTOS ${ }^{1 *}$ \\ ${ }^{1}$ Universidade Federal de Sergipe, Departamento de Ciência e Tecnologia de Alimentos \\ e-mail: joaoantonio@ufs.br
}

\begin{abstract}
RESUMO
A tecnologia de secagem é amplamente utilizada, com a finalidade de aumentar a vida útil dos produtos. A secagem das hortaliças é uma alternativa para redução do desperdício. O objetivo deste trabalho foi avaliar o processo de secagem em uma estufa de secagem com luz de folhas de seis hortaliças: hortelã miúda, coentro, salsa, arruda, tomilho e manjericão. Foram realizadas análises de cor e umidade no tempo inicial da secagem e no tempo final. A secagem foi conduzida em estufa de secagem de lâmpadas a $50^{\circ} \mathrm{C}$. Para obtenção da curva de secagem, realizaram-se pesagens em um intervalo de tempo de 10 minutos durante o período da primeira hora e no intervalo de 20 minutos até alcançar umidade final. Foram utilizados os modelos matemáticos de Hederson e Pabis, Modelo de Newton, Modelo de Page, Modelo Logarítmico e Modelo de Wang e Singh. As equações, de Hederson e Pabis; Newton; Page; Logarítmico e Wang e Singh utilizadas para ajustar o comportamento da cinética de secagem das amostras de hortelã miúda, coentro, salsa, arruda, tomilho e manjericão se mostraram eficientes com altos valores de coeficientes de correlação e podem ser utilizadas para descrever o processo de secagem destas hortaliças na condição de secagem utilizada. Nas condições de secagem estudadas, o tomilho apresentou uma melhor resposta no tocante aos parâmetros de cor, visto que não houve diferença significativa $(\mathrm{p}<0,05)$ na amostra in natura e seca.
\end{abstract}

\section{INTRODUÇÃO}

Hortaliça é a designação da planta herbácea da qual uma ou mais partes podem ser utilizadas como alimento na sua forma natural (ANVISA, 1978). As hortaliças são alimentos altamente perecíveis e apresentam altos índices de perdas pós-colheita. O mercado de hortaliças vem se estruturando em diversos segmentos, apresentando novas tendências de consumo além das hortaliças in natura, como as minimamente processadas, congeladas, conservas, enlatados, desidratadas e liofilizadas (VILELA e HENZ, 2000).

A tecnologia de secagem é amplamente utilizada pelas indústrias, com a finalidade de aumentar a vida útil dos produtos, permitindo também o transporte e o armazenamento sem a cadeia do frio. A remoção de umidade provoca diminuição da atividade de água do produto, inibe o desenvolvimento de microrganismos e retarda deteriorações de origem físico-química (CANO-CHAUCA et al., 2004).

A secagem de alimentos é um fenômeno complexo, que requer representações para a predição do comportamento e otimização de seus parâmetros. Para auxiliar nesta questão, os modelos matemáticos têm sido empregados para estimar o tempo e representar o processo de secagem (SILVA et al., 2008). 
Os métodos teóricos usados para descrever $o$ processo de secagem são baseados em leis físicas que tentam explicar o mecanismo de transferência de água. A principal teoria utilizada para interpretar a secagem de alimentos e de produtos agrícolas é a teoria da difusão da umidade como líquido ou vapor, representada pela segunda lei de Fick (CRANK, 1975).

O objetivo deste trabalho foi avaliar o processo de secagem em uma estufa de secagem com luz de folhas de seis hortaliças: hortelã miúda, coentro, salsa, arruda, tomilho e manjericão; utilizando posteriormente ajustes dos dados experimentais em diferentes modelos e selecionados.

\section{MATERIAIS E MÉTODOS}

\subsection{Obtenção e preparo da amostra}

Como matéria-prima utilizou-se seis hortaliças: hortelã miúda, coentro, salsa, arruda, tomilho e manjericão provenientes do Centro de Abastecimento - CEASA, localizado na cidade de Aracaju - SE. As amostras foram imediatamente transportadas para serem processadas.

As hortaliças foram previamente limpas e lavadas em água corrente, para a remoção de sujidades, sendo, posteriormente, separada as folhas, desprezando o caule e a raiz.

\subsection{Análise de umidade}

Amostras foram coletadas no início e no final do processo de secagem e submetidas ao ensaio de umidade em estufa a $105^{\circ} \mathrm{C}$ até peso constante (IAL, 2004).

\subsection{Análise de cor}

A análise de cor instrumental das amostras de hortaliças, foram avaliadas por meio de leitura direta das folhas com colorímetro da marca Konica Minolta ${ }^{\circledR}$. As coordenadas retangulares foram registradas conforme a CIE (Comission Internatinale de E'clairage), onde $a^{*}$ varia do verde (-) ao vermelho (+), b* do azul (-) ao amarelo (+) e $\mathrm{L}^{*}$ do preto (0) ao branco (100). O ângulo de matiz $\left(h^{\circ}\right)$ é o ângulo do círculo, derivado dos valores de $a^{*}$ e $b^{*}$, que representa a refletância da cor na superfície do material. E a saturação ou cromaticidade (C) é o desvio a partir do ponto correspondente ao cinza no eixo $L^{*}$.

\subsection{Cinética de secagem}

$\mathrm{Na}$ secagem das hortaliças utilizou-se uma estufa de secagem acoplada com duas lâmpadas incandescentes para atingir temperatura de $50^{\circ} \mathrm{C}$. Para obtenção da curva de secagem, realizaram-se pesagens em um intervalo de tempo de 10 minutos durante o período da primeira hora e no intervalo de 20 minutos até alcançar peso constante.

\subsection{Modelagem do processo de secagem}

Os estudos cinéticos foram realizados para determinação das curvas das isotermas de dessorção das hortaliças, ajustando-se aos dados experimentais de secagem, através das Equações abaixo: 1. Hederson e Pabis (HENDERSON e PABIS, 1961); 2. Modelo de Newton (BRUCE, 1985); 3. Modelo de Page (MENGES e ERTEKIN, 2006), 4. Modelo Logarítmico (TOGRUL e PEHLIVAN, 2002) e 5. Modelo de Wang e Singh (WANG et al., 2007)

$$
\begin{aligned}
& R U=\exp (-k t) \\
& R U=a \cdot \exp (-k t) \\
& R U=C \cdot \exp \left(-k t^{n}\right) \\
& R U=C \cdot \exp (-k t)+b \\
& R U=1+a \cdot t+b \cdot t^{2}
\end{aligned}
$$

\subsection{Análise estatística}

O software Statistica ${ }^{\circledR}$ versão 7.0 para Windows foi utilizado para ajustar as equações dos dados experimentais. $\mathrm{O}$ coeficiente de correlação $\mathrm{R}^{2}$ foi utilizado para descrever o melhor ajuste das equações de cinética de secagem. 
O software Assitat versão 7.7 beta, foi utilizado para a realização do teste de Tukey.
As curvas da cinética de secagem das hortaliças estão apresentadas na Figura 1.

\section{RESULTADOS E DISCUSSÃO}

Figura 1 - Conteúdo de umidade adimensional (base seca) versus tempo ajustado para as Equações de Hederson e Pabis; Newton; Page; Logarítmico e Wang e Singh. (A) hortelã miúda, B) coentro, C) salsa, D) arruda, E) tomilho e F) manjericão).
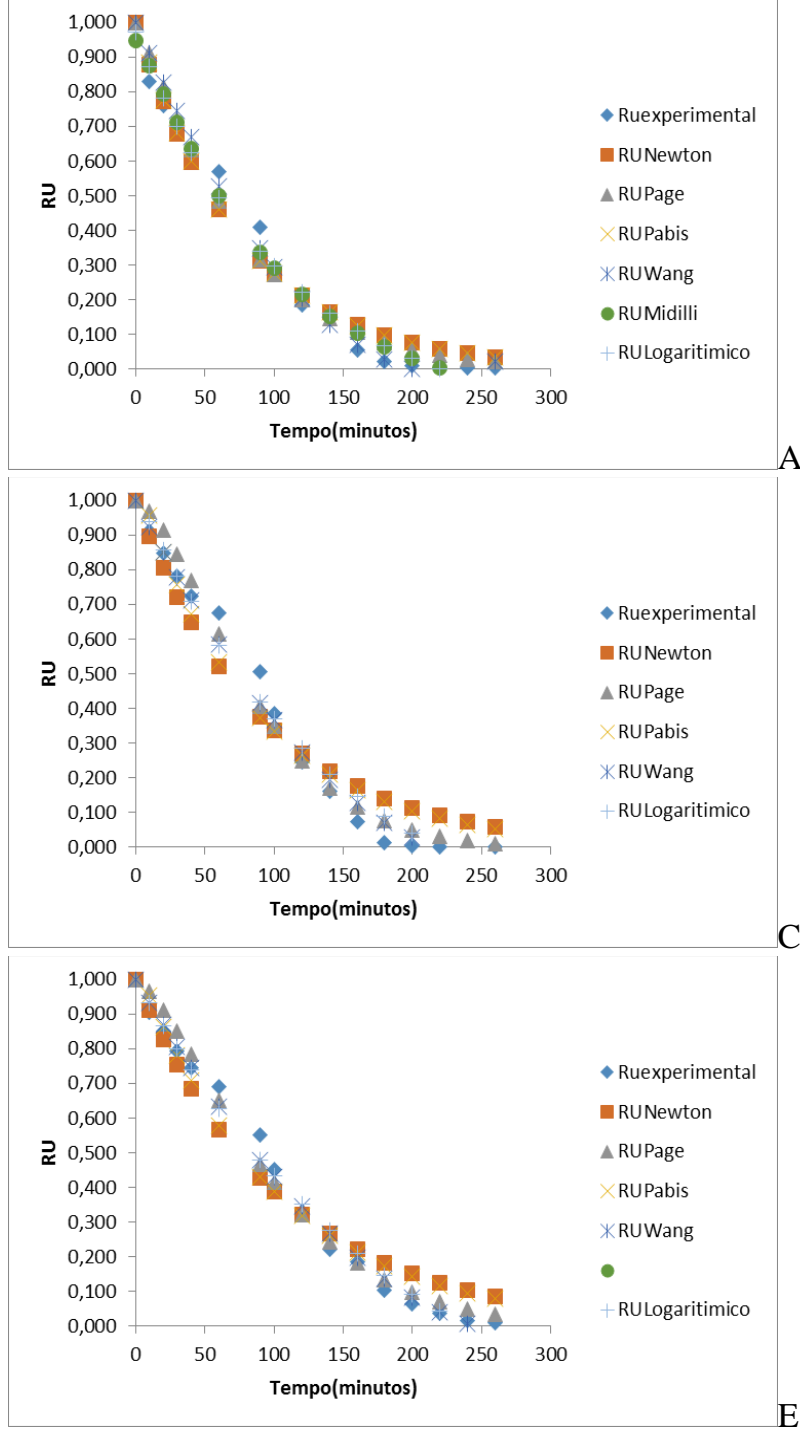

Fonte: Autores (2015).

Ao analisar a Figura 1, pode-se verificar que na temperatura estudada, a razão de umidade reduziu rapidamente no início e, posteriormente, diminuiu lentamente à medida que se aumentava o tempo de secagem. Esses resultados estão de acordo
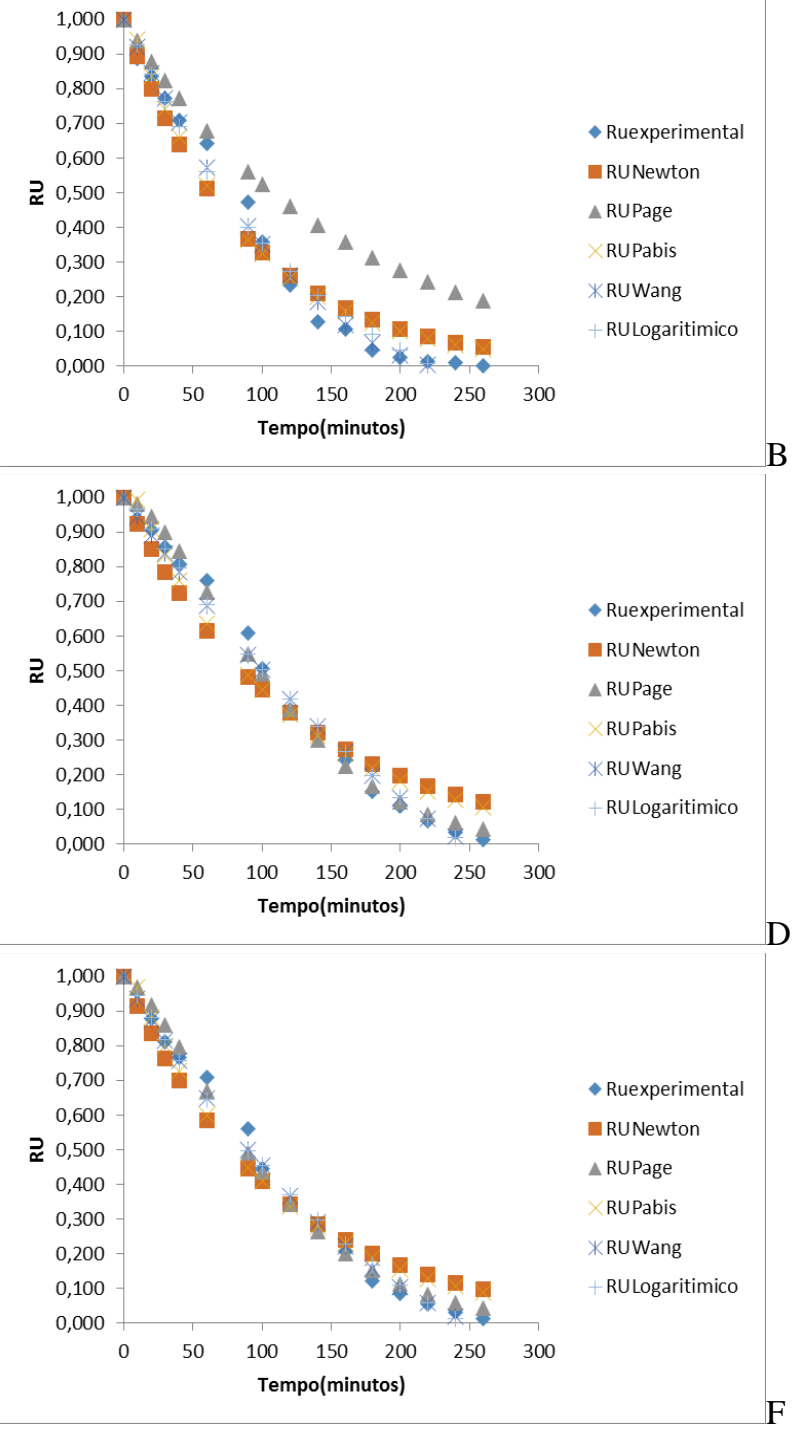

com a literatura (KARABULUT et al., 2007; LAHSASNI et al., 2004).

$\mathrm{Na}$ Tabela 1 encontram-se os parâmetros estatísticos utilizados para a comparação entre os cinco modelos de secagem analisados, nas condições de 
secagem utilizadas para as ervas e o valor das constantes dos modelos.

Para a temperatura utilizada na secagem verifica-se que todos os modelos matemáticos ajustados aos dados experimentais, apresentaram coeficientes de determinação $\left(R^{2}\right)$ superiores a 0,97 , sendo o modelo de Wang et al., (2007) o que apresentou maior valor de $R^{2}$ na temperatura de secagem estudada $\left(50^{\circ} \mathrm{C}\right)$ para todas as hortaliças, com exceção da arruda, onde o modelo que apresentou maior valor de $R^{2}$ foi o modelo de Page (MENGES e ERTEKIN, 2006) (Tabela 1). Quanto maior o $R^{2}$, melhor é o procedimento de ajuste (TOGRUL e PEHLIVAN, 2004; MAZUTTI et al., 2010; SANJINEZ-ARGANDOÑA et al., 2011).

Tabela 1 - Coeficientes de determinação $\mathrm{R}^{2}$ para modelos analisados.

\begin{tabular}{lcccccc}
\hline & $\begin{array}{c}\text { Hortelã } \\
\text { Miúdo }\end{array}$ & Coentro & Salsa & Arruda & Tomilho & Manjericão \\
\hline Newton & 0,98693 & 0,98077 & 0,97276 & 0,97215 & 0,97897 & 0,98048 \\
Page & 0,98933 & 0,99310 & 0,99102 & 0,99625 & 0,99257 & 0,99546 \\
Logarítimico & 0,99284 & 0,99268 & 0,98998 & 0,99529 & 0,99460 & 0,99616 \\
$\begin{array}{l}\text { Wang e } \\
\text { Singh }\end{array}$ & 0,99322 & 0,99590 & 0,99339 & 0,99544 & 0,99601 & 0,99718 \\
$\begin{array}{l}\text { Herderson } \\
\text { Pabis }\end{array}$ & 0,98695 & 0,98302 & 0,97640 & 0,97828 & 0,98130 & 0,98130 \\
\hline
\end{tabular}

Os resultados da influência do método de secagem sobre a cor das folhas das hortaliças estão apresentados na Tabela 2.

Pode-se observar que não houve influência do método de secagem para os parâmetros $a^{*}, C$, e $h^{\circ}$ nas folhas de coentro, tomilho e salsa. Porém, na arruda, hortelã miúdo e manjericão, houve uma redução do valor do parâmetro $a^{*}$, o que indica uma redução da intensidade da coloração verde nas amostras de hortaliças após a secagem e uma redução também na Cromaticidade $(C)$ e na refletância da cor na superfície do material $\left(h^{\circ}\right)$.

Apenas o Tomilho e a Salsa não apresentaram diferença significativa $(\mathrm{p}<0,05)$ no parâmetro $b^{*}$. No que se refere a luminosidade $\left(L^{*}\right)$, todas as amostras apresentaram redução com exceção do tomilho.

Tabela 2 - Valores médios dos parâmetros de cor das ervas in natura $\left(\mathrm{T}_{0}\right)$ e secas $\left(\mathrm{T}_{\mathrm{f}}\right)$.

\begin{tabular}{cccccccc}
\hline & & Coentro & Tomilho & Salsa & Arruda & $\begin{array}{c}\text { Hortelã } \\
\text { Miúdo }\end{array}$ & Manjericão \\
\hline$L^{*}$ & $\mathrm{~T}_{0}$ & $43,86 \pm 1,80 \mathrm{a}$ & $32,16 \pm 4,25 \mathrm{a}$ & $41,82 \pm 3,58 \mathrm{a}$ & $44,56 \pm 2,85 \mathrm{a}$ & $41,20 \pm 0,42 \mathrm{a}$ & $41,23 \pm 6,10 \mathrm{a}$ \\
& $\mathrm{T}_{\mathrm{f}}$ & $32,63 \pm 2,37 \mathrm{~b}$ & $27,20 \pm 0,4 \mathrm{a}$ & $34,36 \pm 2,92 \mathrm{~b}$ & $37,63 \pm 1,26 \mathrm{~b}$ & $32,66 \pm 2,77 \mathrm{~b}$ & $27,03 \pm 1,57 \mathrm{~b}$ \\
$b^{*}$ & $\mathrm{~T}_{0}$ & $36,96 \pm 1,53 \mathrm{a}$ & $16,13 \pm 2,69 \mathrm{a}$ & $25,03 \pm 3,23 \mathrm{a}$ & $26,9 \pm 2,09 \mathrm{a}$ & $30,26 \pm 2,05 \mathrm{a}$ & $26,00 \pm 3,14 \mathrm{a}$ \\
& $\mathrm{T}_{\mathrm{f}}$ & $24,00 \pm 2,70 \mathrm{~b}$ & $12,90 \pm 1,32 \mathrm{a}$ & $23,43 \pm 1,11 \mathrm{a}$ & $22,93 \pm 1,15 \mathrm{~b}$ & $16,60 \pm 0,7 \mathrm{~b}$ & $16,23 \pm 8,35 \mathrm{~b}$ \\
$a^{*}$ & $\mathrm{~T}_{0}$ & $-7,26 \pm 0,96 \mathrm{a}$ & $-2,93 \pm 2,93 \mathrm{a}$ & $-7,5 \pm 0,2 \mathrm{a}$ & $-7,93 \pm 0,41 \mathrm{a}$ & $-8,36 \pm 4,3 \mathrm{a}$ & $-7,66 \pm 0,58 \mathrm{a}$ \\
& $\mathrm{T}_{\mathrm{f}}$ & $-4,23 \pm 2,91 \mathrm{a}$ & $-2,70 \pm 2,7 \mathrm{a}$ & $-5,6 \pm 1,77 \mathrm{a}$ & $-2,23 \pm 0,6 \mathrm{~b}$ & $-0,4 \pm 0,0 \mathrm{~b}$ & $-3,00 \pm 0,34 \mathrm{~b}$ \\
$C^{C}$ & $\mathrm{~T}_{0}$ & $31,16 \pm 9,60 \mathrm{a}$ & $16,46 \pm 3,00 \mathrm{a}$ & $27,16 \pm 3,3 \mathrm{a}$ & $28,06 \pm 2,08 \mathrm{a}$ & $31,23 \pm 2,31 \mathrm{a}$ & $27,1 \pm 3,02 \mathrm{a}$ \\
& $\mathrm{T}_{\mathrm{f}}$ & $24,36 \pm 2,81 \mathrm{a}$ & $13,20 \pm 1,25 \mathrm{a}$ & $23,30 \pm 0,1 \mathrm{a}$ & $23,11 \pm 1,13 \mathrm{~b}$ & $16,6 \pm 0,7 \mathrm{~b}$ & $12,46 \pm 1,53 \mathrm{~b}$ \\
$h^{\circ}$ & $\mathrm{T}_{0}$ & $103,20 \pm 1,41 \mathrm{a}$ & $99,73 \pm 5,38 \mathrm{a}$ & $107,23 \pm 1,85 \mathrm{a}$ & $106,50 \pm 0,65 \mathrm{a}$ & $105,43 \pm 3,96 \mathrm{a}$ & $106,53 \pm 1,96 \mathrm{a}$ \\
& $\mathrm{T}_{\mathrm{f}}$ & $99,96 \pm 1,72 \mathrm{a}$ & $78,06 \pm 2,71 \mathrm{a}$ & $102,2 \pm 3,15 \mathrm{a}$ & $95,60 \pm 1,44 \mathrm{~b}$ & $90,5 \pm 1,56 \mathrm{~b}$ & $76,2 \pm 1,18 \mathrm{~b}$ \\
\hline
\end{tabular}


Fonte: Autor (2015). As médias seguidas pela mesma letra não diferem estatisticamente pelo teste de Tukey a $5 \%$ de probabilidade.

\section{CONCLUSÃO}

As Equações, de Hederson e Pabis; Newton; Page; Logaritimico e Wang e Singh utilizadas para ajustar o comportamento da cinética de secagem das amostras de hortelã miúda, coentro, salsa, arruda, tomilho e manjericão se mostraram eficientes com altos valores de coeficientes de correlação e podem ser utilizadas para descrever o processo de secagem destas hortaliças na condição de secagem utilizada.

Nas condições de secagem estudadas, o tomilho apresentou uma melhor resposta no tocante aos parâmetros de cor, visto que não houve diferença significativa $(\mathrm{p}<0,05)$ na amostra in natura e seca.

\section{NOMENCLATURA}

$\mathrm{a}=$ constante de modelo;

$\mathrm{b}=$ constante de modelo;

$\mathrm{C}=$ constante de modelo;

$\mathrm{k}=$ constante de modelo;

$\mathrm{t}=$ tempo $(\mathrm{min})$;

$\mathrm{R}^{2}=$ coeficiente de determinação;

$\mathrm{RU}=$ umidade relativa;

$\mathrm{T}_{0}=$ tempo inicial;

$\mathrm{T}_{\mathrm{f}}=$ tempo final;

$a^{*}=$ coordenada de cor, varia do verde (-) ao vermelho $(+)$;

$b^{*}=$ coordenada de cor, varia do azul (-) ao amarelo (+);

$L^{*}=$ coordenada de cor, varia do preto $(0)$ ao branco (100);

$h^{o}=$ ângulo de matiz;

$C=$ cromaticidade.

\section{REFERÊNCIAS}

ANVISA - Agência Nacional de Vigilância Sanitária, Comissão Nacional de Normas e
Padrões para Alimentos, Resolução CNNPA n ${ }^{\circ} .12$ - D.O. de 24/07/1978, 1978.

BRUCE, D. M. Exposed-layer barley drying, three models fitted to new data up to $150{ }^{\circ} \mathrm{C}$. Journal of Agricultural Engineering Research, v. 32, n. 4, p. 337-347, 1985.

CANO-CHAUCA, M.; RAMOS, A. M.; STRINGHETA, P. C.; MARQUES, J. A.; SILVA, P. I. Curvas de secagem e avaliação da atividade de água da banana passa.

Boletim Centro de Pesquisa de Processamento de Alimentos, v.22, n.1, p.121-132, 2004.

CRANK, J. The Mathematics of Diffusion. Oxford: Clarendon Press, 1975.

HENDERSON, S. M.; PABIS, S. Grain drying theory I. Temperature effect on drying coefficient. Journal of Agriculture Engineering Research, v. 6, n. 3, p. 169-174, 1961.

IAL. Normas Analíticas do Instituto Adolfo Lutz. 4ed. São Paulo: IMESP, 2004. 1004p.

KARABULUT, I.; HAYALOGLU, A. A.; YILDIRIM, H. Thin-layer drying characteristics of kurut, a Turkish dried dairy by-product. International Journal of Food Science and Technology, v. 42, n. 9, p. 10801086, 2007.

LAHSASNI, $\quad$ S.; $\quad$ KOUHILA, $\quad$ M.; MAHROUZ, M.; JAOUHARI, J. T. Drying kinetics of prickly pear fruit (Opuntia ficus indica). Journal of Food Engineering, v. 61, n. 2, p. 173-179, 2004.

MAZUTTI, M. A.; ZABOT, G.; BONI, G.; SKOVRONSKI, A.; OLIVEIRA, D.; DI LUCCIO, M.; OLIVEIRA, J. V.; RODRIGUES, M. I.; TREICHEL, H.; 
MAUGERI, F. Mathematical modeling of thin-layer drying of fermented and nonfermented sugarcane bagasse. Biomass and Bioenergy, v. 34, n. 5, p. 780-786, 2010.

MENGES, H. O.; ERTEKIN, C. Mathematical modeling of thin layer drying of golden apples. Journal Food Engineering, v. 77, n. 1, p. 119-125, 2006.

SANJINEZ-ARGANDOÑA, E. J.; BRANCO, I. G.; BITTENCOURT, T. U.; MUNHOZ, C; L; Influência da geometria e da temperatura na cinética de secagem de tomate (Lycopersicum esculentum). Ciência e Tecnologia de Alimentos, v. 31, n. 2, p. 308312, 2011.

SILVA, A.S. ALMEIDA, F.A.C. SILVA, F.L.H. DANTAS, H.J. LIMA, E.E. Desidratação e efeito de pré-tratamentos no extrato seco do pimentão verde. Revista Brasileira de Produtos Agroindustriais, v. 10, n. 1, p. 27-34, 2008.

TOGRUL, L. T.; PEHLIVAN, D. Mathematical modeling of solar drying of apricots in thin layers. Journal of Food Engineering, v. 55, n. 3, p. 209-16, 2002.

VILELA, N. J.; HENZ, G.P. Situação atual da participação das hortaliças no agronegócio brasileiro e perspectivas futuras. Cadernos de Ciência e Tecnologia, Brasília, v.17,p.71-89, jan./abril. 2000.

WANG, Z.; SUN, J.; LIAO, X.; CHEN, F.; ZHAO, G.; WU, J.; HU, X. Mathematical modeling on hot air drying of thin layer apple pomace. Food Research International, Toronto, v. 40, p. 39-46, 2007. 